\title{
3D Electromagnetic Positioning Optimization by Means of Deep Learning
}

\author{
H. ÇARK $^{a, *}$, B. BorU ${ }^{b}$ AND A. YAHYA TEŞNELI ${ }^{c}$ \\ ${ }^{a}$ Sakarya University of Applied Science, Graduate Education Institute, Sakarya, Turkey \\ ${ }^{b}$ Sakarya University of Applied Science, Department of Mechatronics Engineering, Sakarya, Turkey \\ ${ }^{c}$ Sakarya University, Department of Electrical and Electronics Engineering Sakarya, Turkey
}

\begin{abstract}
The 3D electromagnetic positioning system consists of four generating coils and three-axis magnetic sensor, accelerometer, and gyroscope in magnetic field. These systems are generally used in navigation, ballistic missile tracking, medicine, robotics, biomechanics, and education. Electromagnetic positioning can be performed in a limited volume. In addition, there are errors in the position calculation. In this study, the aim is to increase the coverage volume and to minimize the errors in the sensor position. Therefore, large radius coil, high circuit current, and high number turn of coil were used to increase the working volume. By optimizing, the sensor was moved closest to the actual position. In order to reduce these errors different software and algorithms were used. Some of them are Levenberg-Marquardt, artificial neural networks, etc. In this study, deep learning algorithms, which are a more advanced version of machine learning concept, are used. Deep networks can be thought of as a special case of multi-layered classical artificial neural networks. Mean square error (MSE) was used for performance analysis of the system.
\end{abstract}

DOI: 10.12693/APhysPolA.137.527

PACS/topics: deep learning, electromagnetic positioning, optimization

\section{Introduction}

The 3D electromagnetic positioning study was carried out for the 3D digitizer and tracking design. Most of the electromagnetic tracking techniques are based on accurate mapping of a 3D magnetic field around generating coils and computing from the field mapped by the sensor position and orientation relative to the source [1]. The operating volume of these systems is directly related to the current supplying transmitter coils, the number of spirals of the coils, the external radius, and the sensitivity of the receiver. In the study, firstly, large radius coil, high circuit current, and high number turn of coil were used to increase the working volume. Then, reducing position errors were aimed. It has been optimized to reduce these errors. Deep learning algorithm is used for position optimization. The mean square error (MSE) values were analysed for performance.

\section{Materials and methods}

In order to test the position information of the 3D positioning, the layout of the coils in the $X-Y$ plane is shown in Fig. 1. The distance between the centres of the transmitting coils in the layout plane was $d=68 \mathrm{~cm}$. In order to increase the operating volume of the system, the outer radius of the transmitter coils was large, the number of spirals was sufficient, and the coil is operated with a high current. There were 243 positions determined

\footnotetext{
*corresponding author; e-mail: hcark32@hotmail.com
}

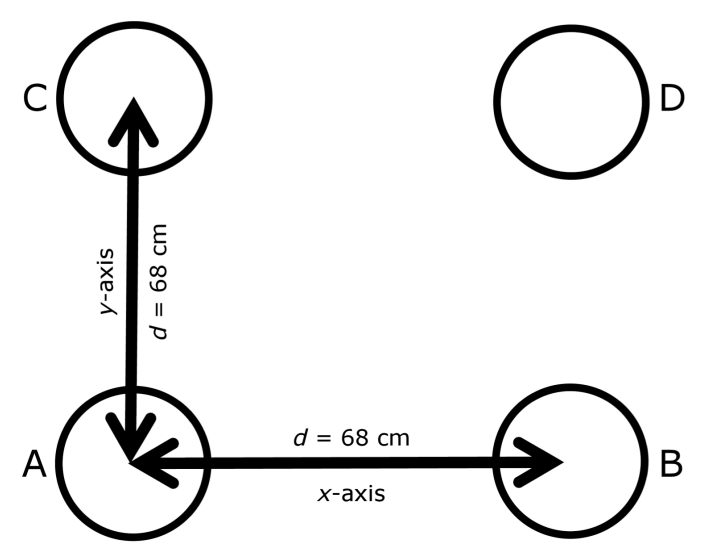

Fig. 1. Layout of transmitter coils in $X-Y$ plane.

for measurement. 81 of the 243 position data obtained were used for training in deep learning algorithm. The positions obtained were calculated according to the point A shown in Fig. 1. The gyroscope and accelerometer were used to determine the pitch and roll angle of the sensor. Coordinate calculations must be made when the sensor transmitter is parallel to the coils.

\subsection{Position parameter and calculation}

The formula for magnetic dipole moment coefficient $k$ is the following:

$$
k=\frac{\mu_{0} \mathrm{~N} \pi R^{2} I}{4 \pi},
$$

where $I$ - current feeding the coil, $N$ - spiral number of the transmitter coil, $R$ - external radius of the transmitter coil, $\mu_{0}$ - magnetic permeability of the gap. 
The positioning formulae are given below [2]:

$$
\begin{aligned}
& B_{A X}=\frac{3 k x z}{r_{A}^{5}} \quad B_{A Y}=\frac{3 k y z}{r_{A}^{5}} \\
& B_{A Z}=\frac{k\left(2 z^{2}-x^{2}-y^{2}\right)}{r_{A}^{5}} \quad r_{A}=\sqrt{x^{2}+y^{2}+z^{2}} \\
& B_{B X}=\frac{3 k(x-d) z}{r_{B}^{5}} \quad B_{B Y}=\frac{3 k y z}{r_{B}^{5}} \\
& B_{B Z}=\frac{k\left(2 z^{2}-(x-d)^{2}-y^{2}\right)}{r_{B}^{5}} \\
& r_{B}=\sqrt{(x-d)^{2}+y^{2}+z^{2}} \\
& B_{C X}=\frac{3 k x z}{r_{C}^{5}} \quad B_{C Y}=\frac{3 k(y-d) z}{r_{C}^{5}} \\
& B_{C Z}=\frac{k\left(2 z^{2}-x^{2}-(y-d)^{2}\right)}{r_{C}^{5}} \\
& r_{C}=\sqrt{x^{2}+(y-d)^{2}+z^{2}} \\
& B_{D X}=\frac{3 k(x-d) z}{r_{D}^{5}} \quad B_{D Y}=\frac{3 k(y-d) z}{r_{D}^{5}} \\
& B_{D Z}=\frac{k\left(2 z^{2}-(x-d)^{2}-(y-d)^{2}\right)}{r_{D}^{5}} \\
& r_{D}=\sqrt{(x-d)^{2}+(y-d)^{2}+z^{2}}
\end{aligned}
$$

\subsection{Deep learning}

Deep learning can be defined as artificial neural networks that contain multiple hidden layers. Unlike basic machine learning approaches, they contain more neurons. They consist of a hierarchically more complex structure than artificial neural networks. Deep learning algorithms consume more power during the training phase [3]. In the use of machine learning techniques, the feature vector must be extracted first. In order to extract the feature vector, experts skilled in the art are needed. These processes take a lot of time involving the expert. For this reason, machine learning techniques cannot process raw data. In contrast to these techniques, deep networks perform learning on raw data. It obtains the necessary information in different layers while processing raw data [4]. Deep learning methods have achieved great suc-

\begin{tabular}{|c|c|c|c|c|c|}
\hline & \multirow{2}{*}{$\begin{array}{l}\text { MSE } \\
\text { value }\end{array}$} & \multirow{2}{*}{$\begin{array}{l}\text { Duration } \\
\text { of training }\end{array}$} & \multicolumn{3}{|c|}{$\begin{array}{l}\text { Avg. axis } \\
\text { errors [mm] }\end{array}$} \\
\hline & & & $X$ & $Y$ & $Z$ \\
\hline $\begin{array}{l}\text { Measurement } \\
\text { results }\end{array}$ & 0.4523 & - & 3.66 & 3.45 & 9.33 \\
\hline $\begin{array}{l}\text { ANN (trainbr, } \\
\text { cascadeforwardnet) }\end{array}$ & 0.3779 & $11.5 \mathrm{~s}$ & 2.91 & 2.64 & 6.71 \\
\hline Deep learning & 0.2191 & $44 \min 35 \mathrm{~s}$ & 3.25 & 3.91 & 4.06 \\
\hline
\end{tabular}
cess in many areas such as finance, health, linguistics and robotics. Modern computer hardware makes it possible to train very large deep neural networks of high dimensions [5].

\section{Experimental results}

In this study, 243 measurements were made in the range of $18-50 \mathrm{~cm}$ and the error values between the real places and the measured places were determined. The
Optimization methods comparison table.

TABLE I

Literature comparison table

TABLE II

\begin{tabular}{l|c|c|c|c}
\hline \hline \multirow{1}{*}{$\begin{array}{c}\text { Studies } \\
\text { in the literature }\end{array}$} & Distance [cm] & \multicolumn{4}{c}{$\begin{array}{c}\text { Axis errors } \\
\text { (average error) [mm] }\end{array}$} \\
\cline { 2 - 5 } $\begin{array}{l}\text { Z. Zhang } \\
\text { and G. Liu [6] }\end{array}$ & 10 & \multicolumn{3}{|c}{$2-4$} \\
\hline $\begin{array}{l}\text { M.N. Islam } \\
\text { and A.J. Fleming [7] }\end{array}$ & 11.7 & 4.8 & 2.4 & 8.8 \\
\hline $\begin{array}{l}\text { M.N. Islam } \\
\text { and A.J. Fleming [7] }\end{array}$ & 25.7 & 3.2 & 2.7 & 12 \\
\hline X. Guo et al. [8] & 30 & $8-24$ & $11-23$ & $9-26$ \\
\hline $\begin{array}{l}\text { C. Du et al. [9] } \\
\text { C. Du et al. [9] }\end{array}$ & $30 \times 30$ & & 6.37 \\
\hline $\begin{array}{l}\text { In this study } \\
\text { (deep learning) }\end{array}$ & $\begin{array}{l}15 \times 30 \\
(18-50)\end{array}$ & 3.25 & 3.91 & 4.06 \\
\hline
\end{tabular}

MSE values were obtained from the measurement results according to the actual positions. Then, artificial neural networks were applied. The best performance was observed in the cascadeforwardnet NN, which was trained by trainbr. Finally, the deep learning algorithm was applied. According to the measurement results, the MSE value was 0.4523 , as shown in Table I, and the MSE value was 0.3779 after the trainbr trained cascadeforwardnet NN was applied. According to the results of deep learning, the value of MSE was 0.2191 and average axis error were $3.24 \mathrm{~mm}$ for $X$-axis, $3.91 \mathrm{~mm}$ for $Y$-axis, and $4.05 \mathrm{~mm}$ for $Z$-axis, as shown in Table I. The duration of training of these algorithms is shown in Table I.

\section{Conclusions}

The 3D electromagnetic positioning systems are generally used in fields such as 3D digitizer, tracking, navigation, biomechanics, robotics, etc. Electromagnetic positioning can be performed in a limited volume. The study was conducted in a range of $X=18-50 \mathrm{~cm}, Y=18-50 \mathrm{~cm}$, and $Z=2.4-6 \mathrm{~cm}$. The outer radius of the transmitter coil was $11.37 \mathrm{~cm}$. The magnetic field in the $11.37-18 \mathrm{~cm}$ range was very high. This high magnetic field was above the sensor's measuring range. Therefore, no measurement was made at this distance. Since the magnetic field weakened, the required measurement could not be performed at a distance of more than $50 \mathrm{~cm}$. 
There are different studies in the literature in the range of $10-30 \mathrm{~cm}$, as shown in Table II. These studies were either performed at very short distances or the axis errors were high. The coverage of this study was wider than all the studies given in Table II and decrease in the axis errors was observed.

\section{References}

[1] S. Song, C. Hu, B. Li, X. Li , Q. Max, H. Meng, IEEE Trans. Magn. 49, (2013).

[2] W.F. Loke, T.Y. Choi, T. Maleki, L. Papiez, B. Ziaie, B. Jung, IEEE Trans. Biomed. Circuits Syst. 4, 223 (2010).

[3] Z. Turgut, Ph.D. Thesis, İstanbul University, İstanbul 2018 .
[4] Ö. İnik, E. Ülker, Gaziosmanpasa J. Sci. Res., 6, 85 (2017).

[5] A. Mikhailova, N.M. Adams, C.A. Hallsworth, F.D.H. Lau, D.N. Jones, in: Proc. Int. Conf. on Smart Infrastructure and Construction (ICSIC), 2019.

[6] Z. Zhang, G. Liu, J. Electromagn. Anal. Appl. 5, 85 (2013).

[7] M.N. Islam, A.J. Fleming, in: Proc. SENSORS, Valencia 2014 IEEE, 2014.

[8] X. Guo, G. Yan, W. He, J. Med. Eng. Technol. 33, $192(2009)$.

[9] C. Du, X. Chen, Y. Wang, J. Li, D. Yu, Sensors, 14, 9961 (2014). 